\title{
Childhood kidney disease in developing countries: Is it a forgotten disease?
}

Kidney disease affects millions of adults and children worldwide. The 11th World Kidney Day was celebrated on 10 March 2016, and was dedicated to childhood kidney diseases. Many children may be at risk of developing kidney disease at an early age, and this year, World Kidney Day aimed to increase awareness around the fact that many adults have had kidney disease since early childhood. The spectrum of kidney disease in children ranges from treatable disorders without long-term sequelae to life-threatening conditions. Children with acute kidney injury (AKI) may develop long-term consequences leading to chronic kidney disease (CKD) later in life. ${ }^{[1]}$ Worldwide, epidemiological information on the incidence and prevalence of paediatric AKI and CKD is limited, often imprecise, and flawed by methodological differences between the various data sources, although increasing in scope. This is particularly pertinent in Africa, where the focus is on communicable diseases with lack of proper documentation and renal registries. ${ }^{[2]}$ As a result there are insufficient data to guide the authorities responsible for resource allocation. This increases the challenges of providing care for children with kidney disease in a resource-limited environment. The rising incidence of kidney diseases in children highlights the need for early detection and healthy lifestyle, starting at birth and continuing into adult life. The main aim of World Kidney Day 2016 was to inform parents, caregivers, young patients, the general public, and policymakers of the importance of the early detection and appropriate management of childhood kidney disease.

\section{The spectrum of renal disease in African children}

There is a dearth of literature on the spectrum and extent of renal disease in African children. This is due to the slow development of the subspecialty, the lack of trained personnel and the fact that highly technical resources such as renal histopathology, dialysis fluids, machines, immunosuppressive drugs and transplantation services are not readily available. The percentage of renal-related admissions to secondary and tertiary hospitals varies widely from 3.5 to $8.9 \%$ among different centres and countries. The most common cause of admission is AKI due to dehydration and septic shock syndromes. ${ }^{[3,4]}$ This has improved owing to the introduction of oral rehydration, but many rural areas still do not have easy access to clean water and sanitation. In South Africa (SA) the three most common causes of AKI in older children are haemolytic uraemic syndrome (35\%), acute tubular necrosis (31\%) and acute glomerulonephritis (GN) (16\%), whereas $39 \%$ of AKI is caused by acute gastroenteritis and nephrotic syndrome in Nigeria, ${ }^{[5]}$ and $26 \%$ was reported to be due to sepsis and malaria. ${ }^{[3]}$ In Ghana, $11 \%$ of AKI reported was as a result of haemoglobinuria from haemoglobinopathies (for example, sickle cell and G-6-phosphates deficiency). ${ }^{[3]}$

The spectrum of chronic glomerular diseases varies in the different geographical regions of the continent, with the epidemiology of infectious agents implicated in its causation varying considerably as one traverses from the most northern regions of Africa to the south. ${ }^{[6]}$ Schistosoma haematobium and Schistosoma mansoni, together with Salmonella typhi, have been reported as the most highly prevalent forms of GN and nephrotic syndrome in Egypt, while a post-infectious type of proliferative nephropathy is seen in Tunisia (northern Africa). ${ }^{[6,7]}$ In the malarial-infested regions of Kenya, Nigeria, Uganda, and parts of Ghana, Plasmodium malariae is the most frequent cause of GN. In Zimbabwe (southern Africa) a post-infectious, diffuse proliferative, exudative form of GN is most often seen ${ }^{[7]}$ Hepatitis B-related nephropathy contributed to a fair percentage as a cause of nephrotic syndrome in black children prior to 1994 in SA. In KwaZulu-Natal (KZN) Province, the rate was $34.3 \%,{ }^{[7]}$ while it was $12 \%$ in Johannesburg (Gauteng Province) ${ }^{[8]}$ but following the introduction of hepatitis B immunisations in 1994, the disease has almost been eradicated with only two sporadic cases seen in the last decade in $\mathrm{KZN} \cdot{ }^{[9]}$ With better screening and treatment for syphilis, congenital syphilis-associated membranous nephropathy has also almost disappeared. The increasing prevalence of HIVrelated nephropathy in the era of limited or no access to treatment was menacing, with HIV causing an increased burden of all diseases, especially severe gastroenteritis leading to dehydration and prerenal AKI. As a result of the severely immunocompromised state, bacterial, viral, fungal and parasitic infections causing septicaemia and septic shock syndromes also contributed to the development of AKI. The use of nephrotoxic drugs such as aminoglycosides, acyclovir and antifungal agents such as amphotericin B further affected kidney function. ${ }^{[10]}$ Studies in the USA have shown the African American population possesses an 18- to 50-fold increased risk for developing HIV-1-associated focal segmental glomerulosclerosis (FSGS). ${ }^{[11]}$ Significant evidence of the influence of genetic factors on this disparity is that individuals of African ancestry from other geographical regions also show an increased risk for developing FSGS. ${ }^{[12]}$ Fortunately early treatment with highly active antiretroviral therapy and the prevention of mother-to-child treatment programmes, together with screening for proteinuria and haematuria at HIV treatment centres has made an impact on the prevalence of HIV-associated nephropathy with a massive reduction in the incidence of the disease. Autoimmune diseases such as systemic lupus erythematosus and systemic vasculitis, e.g. Takayasu's arteritis, which are occasionally seen in children and have a good prognosis, need to be recognised and referred early. ${ }^{[13]}$

Congenital abnormalities of the kidney and the urinary tract, which include the obstructive uropathies, can be detected with a prenatal sonar. Obstructive uropathy is more common than reflux nephropathy in black African children. Over a 19 -year period at Chris Hani Baragwanath Academic Hospital, Johannesburg, SA, only 21 of 2159 cases $(<1 \%)$ of documented urinary tract infections (UTIs) had primary vesicoureteric reflux (Kala - personal communication). The prevalence of posterior urethral valves and pelviureteric junction obstruction (unilateral or bilateral) is common; some are associated with hypoplastic and dysplastic kidneys that carry a very poor prognosis. An important aspect of minimising renal damage in obstructive uropathy is the availability of prenatal ultrasound in pregnant women. Most mothers in the public health sector do not have access to ultrasonography and are therefore diagnosed late, usually after a UTI, thus compromising kidney function further. ${ }^{[4]}$ One also needs to be alert to the possibility of metabolic and genetic diseases such as renal tubular acidosis, cystinosis, and oxalosis that are sometimes seen in the black African population. The high incidence of premature and small for gestational age (SGA) births in developing countries as a risk factor for kidney disease in adulthood, in the absence of evident kidney disease in early life, has been explained by the Barker hypothesis. ${ }^{11,15]}$ Steps need to be taken to decrease the premature birth rate by educating mothers to book early for antenatal screening, encourage proper nutrition and monitoring by health personnel on the progress of the pregnancy, thus preventing eventual hypertension, metabolic syndrome and possible type 2 diabetes mellitus. ${ }^{[16]}$ It is therefore imperative to follow 
kidney function and blood pressure throughout life in premature and SGA babies. Also supplementing folic acid to potential mothers will decrease spinal dysraphism and lower urinary tract abnormalities.

\section{Transition from paediatric to adult renal services}

The transitioning of children from paediatric to adult services is inadequate due to patient load and poorly trained staff. This leads to poor compliance and loss of an already vulnerable adolescent patient to follow up. It is vital that there is good communication between paediatric and adult services for effective patient handover. Support for this age group of patients should be developed on both sides. If there is familial or societal instability or a disease crisis, transition may need to be slowed, paused, or even temporarily reversed. ${ }^{[17]}$

\section{Call for action}

In developing countries, infections and other non-communicable diseases such as cardiovascular disease, diabetes, cancer, and chronic respiratory disorders, eclipse resource allocation for kidney diseases. Efforts need to be focused on increasing education and awareness on the impact of kidney diseases as a multiplier disease on infections and non-communicable diseases. There must be a greater focus on early detection and management of kidney disease in childhood by health authorities. This is consistent with the objectives of the World Health Organization to reduce mortality from non-communicable diseases with a 10 -year target population level initiative focusing on changes in lifestyle (including tobacco and alcohol use reduction, salt intake control and dietary energy control), and effective interventions (including blood sugar, cholesterol and blood pressure control).

\section{Rajendra Bhimma}

Department of Paediatrics and Child Health, School of Clinical Medicine, Nelson R Mandela School of Medicine, University of KwaZulu-Natal, Durban, South Africa

bhimma@ukzn.ac.za

\section{Udai Kala}

Department of Paediatrics, Faculty of Health Sciences, University of the Witwatersrand, Johannesburg, South Africa
1. Goldstein SL. Acute kidney injury in children and its potential consequences in adulthood. Blood Purif 2012;33(1-3):131-137. DOI:10.1159/000334143

2. Ladapo TA, Esezobor CI, Lesi FE. Pediatric kidney diseases in an African country: Prevalence, spectrum and outcome. Saudi J Kidney Dis Transpl 2014;25(5):1110-1116. DOI:10.4103/1319-2442.139976

3. Antwi SSA, Amoah A, Appia AS, Obeng E. Acute kidney injury in children: 3 year data review from Ghana. Int J Pediatr Res 2015;1:2.

4. Elzouki AY, Amin F, Jaiswal OP. Prevalence and pattern of renal disease in eastern Libya. Arch Dis Child 1983;58(2):106-109.

5. Choopa MS, van Biljoen G. Acute kidney injury in children - not just for the nephrologist. S Afr Fam Pract 2015;2015(57):30-33.

6. Seggie J, Davies PG, Ninin D, Henry J. Patterns of glomerulonephritis in Zimbabwe: Survey of disease characterised by nephrotic proteinuria. Q J Med 1984;53(209):109-118. DOI: 10.1007/BF00869837

7. Bhimma R, Coovadia HM, Adhikari M. Nephrotic syndrome in South African children: Changing perspectives over 20 years. Pediatr Nephrol 1997;11(4):429434. DOI: $10.1007 / \mathrm{s} 004670050310$

8. Kala U, Jacobs DWC, Meyers KEC, Thomson PD. Nephrotic syndrome in children at the Baragwanath and Johannesburg Hospitals: 1982-1998 Inclusive. Kidney Int 1991;39:359.

9. Bhimma R, Coovadia HM, Adhikari M, Connolly CA. The impact of the hepatitis B virus vaccine on the incidence of hepatitis B virus-associated membranous nephropathy. Arch Pediatr Adolesc Med 2003;157(10):10251030. DOI:10.1001/archpedi.157.10.1025

10. Bhimma R, Purswani MU, Kala U. Kidney disease in children and adolescents with perinatal HIV-1 infection. J Int AIDS Soc 2013;16:18596. DOI:10.7448/ IAS.16.1.18596

11. Eggers PW, Kimmel PL. Is there an epidemic of HIV Infection in the US ESRD program? J Am Soc Nephrol 2004;15(9):2477-2485.

12. Kitiyakara C, Kopp JB, Eggers P. Trends in the epidemiology of focal segmental glomerulosclerosis. Semin Nephrol 2003;23(2):172-182. DOI:10.1053/ snep. 2003.50025

13. Faller G, Thomson PD, Kala U, Hahn D. Demographics and presenting clinical features of childhood systemic lupus erythematosus. S Afr Med J 2005;95(6):424-427. DOI:10.1080/22201009.2005.10872120

14. Barker DJ. Fetal origins of cardiovascular disease. Ann Med 1999;31 Suppl 1:3-6.

15. Hoy WE, Ingelfinger JR, Hallan S, Hughson MD, Mott SA, Bertram JF. The early development of the kidney and implications for future health. J Dev Orig Health Dis 2010;1(4):216-233. DOI:10.1017/S204017441000022X

16. Hoy WE, Rees M, Kile E, Mathews JD, Wang Z. A new dimension to the Barker hypothesis: Low birthweight and susceptibility to renal disease. Kidney Int 1999;56(3):1072-1077. DOI:10.1046/j.1523-1755.1999.00633.x

17. Watson AR, Harden P, Ferris M, Kerr PG, Mahan J, Ramzy MF. Transition from pediatric to adult renal services: A consensus statement by the International Society of Nephrology (ISN) and the International Pediatric Nephrology Association (IPNA). Pediatr Nephrol 2011;26(10):1753-1757. DOI:10.1007/s00467-011-1981-z

S Afr J Child Health 2016;10(2):103-104. DOI:10.7196/SAJCH.2016.v10i2.1144 